\title{
Bone mineral status and metabolism in patients with Williams-Beuren syndrome
}

\author{
Stefano Stagi, ${ }^{1}$ Cristina Manoni, ${ }^{1}$ Perla Scalini, ${ }^{1}$ Francesco Chiarelli, ${ }^{2}$ Alberto Verrotti, ${ }^{3}$ \\ Cecilia Cecchi ${ }^{1}$, Elisabetta Lapi, ${ }^{4}$ Sabrina Giglio, ${ }^{4}$ Silvia Romano, ${ }^{4}$ Maurizio de Martino ${ }^{1}$ \\ ${ }^{1}$ Department of Health Sciences, University of Florence, Anna Meyer Children's University Hospital, Florence; ${ }^{2}$ Department \\ of Paediatrics, University of Chieti, Chieti; ${ }^{3}$ Department of Paediatrics, University of L'Aquila, L'Aquila; ${ }^{4}$ Genetics and \\ Molecular Medicine Unit, Anna Meyer Children's University Hospital, Florence; Italy
}

\begin{abstract}
OBJECTIVE: To evaluate bone mineral status and metabolism in a cohort of patients with Williams-Beuren syndrome (WBS). PATIENTS: Thirty-one children (15 females, 16 males; mean age 9.6 \pm 2.74 years) and 10 young adults (6 females, 4 males; mean age $21.4 \pm 5.11$ years) with WBS were cross-sectionally evaluated and compared with two age-, sex-, and body-sizematched paediatric ( 155 subjects, 75 females and 80 males; mean age $9.7 \pm 2.93$ years) and adult (50 subjects, 30 females and 20 males; mean age $22.3 \pm 5.42$ years) healthy controls. MEASUREMENTS: We evaluated ionised and total calcium, phosphate, parathyroid hormone (PTH), 25-hydroxyvitamin $D, 1,25$-dihydroxyvitamin $D$, osteocalcin, bone alkaline phosphatase levels, and urinary deoxypyridinoline concentrations. We also calculated the phalangeal amplitudedependent speed of sound (AD-SoS) and the bone transmission time (BTT) z-scores. RESULTS: WBS patients showed a significantly reduced AD-SoS z-score $(p<0.001)$ and BTT $z$-score $(p<0.001)$ compared with the controls. This finding persisted when we divided the sample into paediatric and adult patients. WBS patients also had significantly higher ionised $(p<0.001)$ and total calcium $(p<0.001)$ levels as well as higher PTH levels $(p<0.001)$ compared with the controls. Furthermore, WBS children and adolescents had significantly lower serum osteocalcin levels $(p<0.001)$ and urinary deoxypyridinoline concentrations $(p<0.001)$ than controls. CONCLUSIONS: WBS subjects exhibit a significant reduction in bone mineral status and impaired bone metabolism. These findings point to the need for close monitoring of WBS patients.
\end{abstract}

Key words: Bone Metabolism, Bone quality, Osteoporosis, QUS, Williams-Beuren syndrome, Williams syndrome

Address for correspondence:

Dr Stefano Stagi, MD, Department of Health Sciences,

University of Florence, Anna Meyer Children's University

Hospital, viale Pieraccini 24, Florence, Italy;

Tel.: +39-055-5662405, Fax: +39-055-5662400,

E-mail: stefano.stagi@yahoo.it

Received: 16-02-2016, Accepted: 31-05-2016

\section{INTRODUCTION}

Williams-Beuren syndrome (WBS; OMIM 194050) is a genetic multisystemic disorder caused by a hemizygous microdeletion on chromosome $7 .{ }^{1}$ The estimated prevalence ranges from $1: 10,000$ to $1: 15,000$ live births. ${ }^{2}$ WBS is characterised by cardiovascular disease, 
distinctive facies and personality, mild intellectual disability, connective tissue abnormalities, growth retardation, and endocrine disorders. ${ }^{3-5}$

Among the endocrine abnormalities associated with WBS, the most frequently noted are short stature, early puberty and early menarche, thyroid diseases, diabetes mellitus, ${ }^{4,5}$ and hypercalcaemia, which has received the most attention. ${ }^{1}$ Hypercalcaemia has been reported in $5-50 \%$ of WBS patients and is generally mild and asymptomatic or associated with nonspecific symptoms. ${ }^{1}$

The causes of hypercalcaemia in WBS are unknown; however, the proposed mechanisms causing hypercalcaemia include vitamin D sensitivity, increased 1,25 dihydroxyvitamin D levels, and defective calcitonin synthesis or release. ${ }^{1,6,7}$

Despite the multiple endocrine, cardiovascular, and gastroenterologic problems reported in these patients, studies looking at metabolism and bone quality are virtually absent from the literature. In a small clinical study conducted on $20 \mathrm{WBS}$ adults of at least 30 years of age, the authors reported 12 individuals meeting criteria for either osteoporosis or osteopoenia at the femoral site, and 10 individuals meeting criteria for either osteoporosis or osteopoenia at the lumbar site. However, this study did not report extensive data about the patients' bone density and metabolism. ${ }^{8}$

Therefore, the purpose of our study was to evaluate bone quality and metabolism in a cohort of children, adolescents, and young adults with WBS.

\section{SUBJECTS AND METHODS}

Forty-one patients ( 21 females and 20 males; mean age $12.5 \pm 6.11$ years) with WBS were recruited consecutively from December 2012 until February 2015 at the Genetics and Molecular Medicine Unit at Anna Meyer Children's University Hospital in Florence, Italy. The cohort included both children (31 patients, 15 females, 16 males; mean age $9.6 \pm 2.74$ years) and young adults (10 patients, 6 females, 4 males; mean age $21.4 \pm 5.11$ years).

Our study was conducted according to the Declaration of Helsinki and the European Guidelines on Good Clinical Practice. Ethical approval was obtained from the Ethics Committee of the Meyer Children's University Hospital. Written informed consent was obtained from parents of the WBS patients. Due to low reading ability and IQ, all participants with WBS provided verbal or written consent after a brief description of the study, according to age (in the case of patients aged $\geq 7$ years) and ability to complete the written consent.

\section{Case definition and study protocol}

WBS diagnosis was made according to clinical phenotype assessed by experienced medical geneticists. The classical phenotype included typical facial features, mental retardation/developmental delay, supravalvular aortic stenosis, other congenital heart disease, inguinal hernia, and hypercalcaemia, as previously reported. ${ }^{5,9}$ Diagnosis was confirmed by fluorescent in situ hybridisation results for elastin deletion at $7 \mathrm{q} 11.23$. All subjects with WBS in this study exhibited the typical $1.55 \mathrm{Mb}$ microdeletion. A minimum of 10 metaphases were scored for the deletion of the $7 q 11.23$ region in each patient. In some cases, array CGH (44 K array platform Agilent oligonucleotides with a resolution of approximately $100 \mathrm{~kb}$ ) was performed to analyse and precisely map the position of each deletion in this region and confirm the minimal region of loss of $1.55 \mathrm{Mb}$.

For the WBS patients and controls, the participants or their parents were asked to fill out a questionnaire that was then reviewed by the medical staff during the baseline examination. The questions were related to the following items: presence or absence of congenital or acquired cardiac defects, a positive history of hypo-/hypercalcaemia, diabetes, thyroid diseases, coeliac disease, current and past medications, especially vitamin $\mathrm{D}$ and/or calcium intake, familial and personal bone fracture history and osteoporosis, and physical activity.

Using an activity questionnaire, physical activity was assessed with a modified activity score composed of scores for sports/leisure activities $(0,<2$ or $>2$ hours per week). Outdoor exposure was quantified from both questions regarding each subject's average number of daily outdoor hours across each season and a prospective daily time-activity diary, as previously described. ${ }^{10}$ 
Calcium dietary and vitamin D intake was assessed using the semiquantitative validated Food Frequency Questionnaire, as previously reported. ${ }^{10,11}$ The selection of items was based on the patient's diet, frequency of eating, and relative importance of food items, such as a calcium and vitamin $\mathrm{D}$ source. The questionnaire included the following food items: milk and dairy products, including calcium-enriched items such as yoghurt, cheese, and chocolate as well as eggs, meat, fish, cereals, bread, vegetables, and fruits.

For all subjects, we determined clinical and demographic data including height, weight, body mass index (BMI), pubertal staging, and, when appropriate, bone age.

The patients were divided into two groups according to their growth velocity, pubertal staging, and/or bone age: 1) paediatrics (patients who had not reached adult height) and 2) adults (patients who had reached adult height). ${ }^{10}$

Exclusion criteria for both WBS patients and controls included already-known bone metabolic diseases, hyper-/hypothyroidism, hyper-/hypoparathyroidism, malabsorptive disorders, chronic renal insufficiency, cancer, and drug addiction on the basis of medical history and questionnaires for osteoporosis risk factors. None of the participants had a recent history of travelling to warmer, sunnier areas prior to and/or during the study and no subjects used any medication that could compromise the study evaluation such as topic or systemic glucocorticoids, anticonvulsant therapy, calcium, vitamin D supplements in the past six months, sexual steroids, or GnRH analogues.

WBS patients and controls underwent a quantitative bone ultrasonometry (QUS) scan to evaluate their bone mineral status. The investigation also consisted of a fasting blood sampling that was analysed to determine the following measurements: serum concentrations of creatinine, albumin, calcium (total and ionised), phosphate, 25-hydroxyvitamin D (25[OH]D), and 1,25-dihydroxyvitamin $\mathrm{D}(1,25[\mathrm{OH}] 2 \mathrm{D})$, plasma levels of parathyroid hormone (PTH), and markers of bone formation (bone alkaline phosphatase and osteocalcin) and bone resorption (urinary deoxypyridinoline). Because PTH-related peptide (PTHrP) shares Nterminal homology with $\mathrm{PTH}$, to exclude any possible interference regarding the PTH assay by the PTHrP, the samples of few WBS subjects ( 3 children and 2 adults) were tested for cross-reactivity with PTHrp; all results were negative for such a cross-reactivity.

Serum 25(OH)D levels were stratified according to the following brackets: $\leq 10,11-20,21-30$, and $>30$ $\mathrm{ng} / \mathrm{mL}$, and defined as severe deficiency, deficiency, insufficiency, and sufficiency, respectively, according to previously established guidelines for bone health (in the absence of a consensus regarding appropriate levels for endocrine and extra-endocrine health). ${ }^{12,13}$

\section{Control group}

The data obtained were compared with two age-, sex-, and body-size-matched healthy control groups for paediatric ( 155 subjects, 75 females and 80 males; mean age $9.7 \pm 2.93$ years) and adult patients ( 50 subjects, 30 females and 20 males; mean age $22.3 \pm 5.42$ years). For every patient, we selected five control subjects who matched the following criteria: age \pm 12 months, height $\pm 5 \mathrm{~cm}$, weight $\pm 2.0 \mathrm{~kg}$, and equivalent pubertal stage. Informed consent was obtained from all subjects and/or parents. These healthy control groups were randomly selected from a population survey of healthy Caucasian inhabitants in Tuscany with no endocrine or metabolic diseases, some of whom were evaluated for non-inflammatory musculoskeletal complaints. Some of the individuals in this group have been previously reported. ${ }^{14}$

\section{QUS scans}

Bone mineral density (BMD) was also evaluated with a DBM Sonic 1200 device (IGEA Bone Profiler, Carpi, Italy). The device was equipped with two probes mounted on an electronic calliper. The emitter probe was positioned on the medial surface and the receiver probe was positioned on the lateral side of the measured phalanx, as previously reported. ${ }^{14}$ The time interval between emission and reception of the ultrasound signal was measured and expressed in $\mathrm{m} / \mathrm{s}$. Using this technique, we measured the amplitudedependent speed of sound (AD-SoS, $\mathrm{m} / \mathrm{s}$ : the interval between the start time of the transmitted signal and the time the signal received reached the predetermined minimum amplitude value of $2 \mathrm{mV}$ for the first time) and the bone transmission time (BTT, $\mu$ s: the difference between the transmission time in the phalanx soft tissue and the bone and transmission time in the 
phalanx soft tissue). AD-SoS and BTT standard deviation score (SDS) were automatically generated. ${ }^{14}$

The final result is the average AD-SoS and BTT over four fingers (digits II-V). All of the QUS measurements were carried out by the same operator on the patients' non-dominant hands. The coefficients of precision, in vivo, were $0.8 \%$ and $1.5 \%$ for $\mathrm{AD}-\mathrm{SoS}$ and BTT, respectively.

Since bone size could influence QUS parameters, ${ }^{15}$ we created a height-adjusted z-score for AD-SoS using control children's data to overcome this bias. Low bone mineral status was defined in the presence of at least one densitometric parameter $\leq-2.0 \mathrm{Z}$-score among AD-SoS or BTT.

\section{Study and laboratory methods}

Height was measured using a wall-mounted stadiometer and weight was measured to the nearest 0.1 $\mathrm{kg}$. All of the measurements were carried out by the same trained staff members. The coefficient of variation $(\mathrm{CV})$ values was $<1 \%$ for these measurements.

We calculated BMI as weight in kilograms divided by height in metres squared $\left(\mathrm{kg} / \mathrm{m}^{2}\right)$. Age-related reference values for height, weight, and BMI were obtained from specific WBS charts ${ }^{16-18}$ for WBS patients and from a wide sample of Italian children for healthy controls. ${ }^{19}$

Height and BMI were normalised for chronological age by converting to SDS. SDS values were calculated according to the following formula: (patient value mean of age-related reference value)/standard deviation of the age-related reference value. ${ }^{20}$ Pubertal staging was carried out according to Tanner and Whitehouse's criteria using an orchidometer for the boys. ${ }^{21}$

All laboratory measurements were performed on blood samples collected after an overnight fast and on a 24-hr urinary collection. Serum levels of calcium, phosphate, creatinine, and albumin were performed at the Clinical Laboratory of the Anna Meyer Children's University Hospital and were measured in all samples by a standard autoanalyser method routinely used for daily practice (Siemens Dimension Vista ${ }^{\circledR}$ 500 Systems, Siemens Healthcare Diagnostics, Tarrytown, NY).

Normal serum concentrations of total calcium are $2.2-2.7 \mathrm{mmol} / \mathrm{L}$ for children and $2.2-2.6 \mathrm{mmol} / \mathrm{L}$ for adults, as previously described. ${ }^{10}$ Normal blood concentrations of phosphate are 1.4-1.7 mmol/L for children aged 2-12 years and 1.1-1.4 $\mathrm{mmol} / \mathrm{L}$ for youth aged 12-16 years of age; the normal adult range is $0.8-1.5 \mathrm{mmol} / \mathrm{L} .^{10}$

Blood-ionised calcium concentrations were measured within a few minutes of sampling with an ICA Kit (McLendon Clinical Laboratories, Chapel Hill, $\mathrm{NC}$, USA). The normal range is $1.10-1.32 \mathrm{mmol} / \mathrm{L}$.

Serum 25(OH)D and PTH were determined by chemiluminiscence enzyme-labeled immunometric assays using an IMMULITE 2000 Systems analyser (Siemens, Gwynedd, UK). The intra- and inter-assays CVs were $<5 \%$ and $<8 \%$ and $<8 \%$ and $<10 \%$, respectively). The normal range is stated as $12.0-72.0 \mathrm{pg} /$ $\mathrm{mL}$. When carried out, PTHrP levels were measured immunoradiometrically (Nichols Institute Diagnostics, San Juan Capistrano, CA, USA).

Serum $1,25[\mathrm{OH}] 2 \mathrm{D}$ was determined according to a competitive binding protein assay (Nichols Institute Diagnostics, San Juan Capistrano, CA, USA). The inter-assay $\mathrm{CV}$ was $8 \%$ and the normal range is stated as $19.9-67.0 \mathrm{pg} / \mathrm{mL}$.

A commercially available radioimmunoassay kit was used to measure serum osteocalcin levels (CIS Diagnostici S.p.A., Tronzano Vercellese, Italy). The sensitivity of the method was $0.50 \mathrm{ng} / \mathrm{mL}$.

Urinary deoxypyridinoline concentrations were measured using high-resolution chromatography in a fluid environment (Medical System, Genova, Italy). We expressed deoxypyridinoline values in $\mathrm{nM}$ for $\mathrm{mM}$ of nocturnal 12-hr urinary creatinine. The intra- and inter-assay CV of RIA and IRMA methods were $<9.8 \%$.

The serum level of bone-specific alkaline phosphatase (BSAP) was measured by immunoassay (Metra Biosystems, Mountain View, CA, USA) with a sensitivity of $0.7 \mathrm{U} / \mathrm{L}$ and a $\mathrm{CV}$ of $3.9-5.8 \%$.

\section{Statistical analysis}

Statistical analyses were performed using SPSSX (SPSSX Inc., Chicago, IL, USA). Summaries of continuous variables are given as mean \pm standard deviation (or median and range, depending on whether 
the data were normally distributed or not). To compare differences, we used the Student's $t$-test and the Mann-Whitney $U$ test depending on the distribution of the analysed variable. The chi-squared test and Fisher's exact test were used to examine associations between dichotomous variables. Spearman's (rank) correlation test was used to determine the correlation coefficients. We used multiple stepwise regression to determine the variables (age [years], sex [M:F], serum PTH concentrations, ionised and total calcium, phosphate, BSAP, 25[OH]D and 1,25[OH]2D levels, serum osteocalcin levels, urinary deoxypyridinoline concentrations, quantitative assessment of physical activity [hours per week], calcium intake [mg/day], and vitamin D intake [I.U./day]) that might correlate independently with $\mathrm{AD}$-SoS z-score values. P values $<0.05$ were considered to be statistically significant.

\section{RESULTS}

The primary demographic, clinical, and laboratory characteristics of patients with WBS and the controls are summarised in Table 1.

The history of fractures was not statistically different between WBS patients ( 2 males and 3 females; $12.2 \%$ ) and the controls ( 8 males and 13 females, $10.2 \%$ ) (Table 1). In addition, no significant differences were found in dietary calcium and vitamin D intakes between the WBS patients and the controls (Table 1).

WBS patients showed a significantly impaired bone mineral status, exhibiting reduced AD-SoS and BTT $\mathrm{z}$-scores compared with controls ( $\mathrm{p}<0.001)$ (Table 1 ). They also showed an impaired bone mineral status compared to healthy controls even when divided into two groups, paediatric $(\mathrm{p}<0.001)$ and adult patients $(\mathrm{p}<0.001)$ (Table 1).

Overall, $51.2 \%$ of WBS patients had a low bone mineral status (z-score $\leq-2.0$ ), which is significantly higher than controls $(2.9 \% ; \mathrm{p}<0.001)$. In particular, AD-SoS was $\leq-2.0 \mathrm{z}$-score in $48.8 \%$ of WBS vs. $2.9 \%$ of controls ( $<<0.001$ ), and BTT $\mathrm{z}$-scores were $\leq-2.0$ in $17.1 \%$ vs. $1.5 \%$ (p $<0.001)$, respectively. Interestingly, compared to the controls, these data maintained the statistical significance when WBS subjects were divided in two groups, children (AD-
SoS z-score $\leq-2.0: 45.1 \%$ vs. $1.9 \%, \mathrm{p}<0.001$; BTT $\mathrm{z}$-score $\leq-2.0: 19.3 \%$ vs. $0.6 \%, \mathrm{p}<0.001)$ and adults (AD-SoS z-score $\leq-2.0$ : $70 \%$ vs. $6.0 \%, \mathrm{p}<0.001$; BTT $z$-score $\leq-2.0$ : $10 \%$ vs. $4 \%, \mathrm{p}=0.002$ ).

Regarding only WBS patients, we disclosed a significantly increased percentage of adults showing a reduced bone mineral status $(70.3 \%$ and $19.3 \%)$ by comparison with the children (45.1\% and $10.1 \%$; respectively, $\mathrm{p}<0.001$ and $\mathrm{p}=0.002$ ). Moreover, WBS patients with a positive history of fracture more frequently show AD-SoS and BTT z-scores $\leq-2.0(80.0 \%)$ compared with WBS patients with a negative history of fracture (respectively, $44.4 \%$ and $8.3 \% ; \mathrm{p}<0.001$ ).

The assessment of bone metabolism enabled us to demonstrate that WBS subjects showed significantly higher ionised $(\mathrm{p}<0.001)$ and total calcium levels $(p<0.001)$ and reduced phosphate levels $(p=0.010)$ compared with controls (Table 1). Interestingly, WBS patients disclosed significantly higher PTH levels compared with controls ( $\mathrm{p}<0.001)$. However, ionised and total calcium and PTH levels did not differ between children and adults with WBS.

Children and young adults with WBS exhibited significantly lower serum osteocalcin levels ( $p$ $<0.001$ ) and urinary deoxypyridinoline concentrations ( $p<0.001)$ compared with age-matched controls. Nonetheless, similar results were not observed when the adult cohorts were compared in terms of osteocalcin ( $10.1 \pm 4.35$ vs. $9.2 \pm 3.97 \mathrm{ng} / \mathrm{mL} ; \mathrm{p}=0.526)$, whereas we discovered a significantly higher urinary deoxypyridinoline concentration in WBS adults compared with controls $(30.1 \pm 5.17 \mathrm{vs} .12 .3 \pm 4.47 \mathrm{nM} / \mathrm{mM}$ creatinine; $p<0.001)$. Furthermore, WBS patients did not exhibit significantly different serum BSAP levels compared with age-matched controls $(100.9 \pm 29.83$ vs. $103.2 \pm 33.51 \mathrm{U} / \mathrm{L}, \mathrm{p}=0.683$ ), whereas a significant difference was discovered comparing WBS adults subgroup with controls ( $76.4 \pm 20.6$ vs. $19.1 \pm 9.3 \mathrm{U} / \mathrm{L}$; $\mathrm{p}<0.001$ ).

None of WBS patients exhibited 25[OH]D values $<10 \mathrm{ng} / \mathrm{mL}$ (deficient). Fifteen patients $(36.6 \%)$ had values of $10-20 \mathrm{ng} / \mathrm{mL}, 18$ patients $(43.9 \%)$ had values of $20-30 \mathrm{ng} / \mathrm{mL}$, and 8 patients $(19.5 \%)$ had values $>30 \mathrm{ng} / \mathrm{mL}$. The mean level of all patients was $23.7 \pm 7.30 \mathrm{ng} / \mathrm{mL}$ (controls: $31.8 \pm 13.24 \mathrm{ng} / \mathrm{mL}$; $\mathrm{p}<0.001)$. Additionally, $1,25[\mathrm{OH}] 2 \mathrm{D}$ values were in 
Table 1. Baseline characteristics of Williams-Beuren syndrome (WBS) patients and controls

\begin{tabular}{|c|c|c|c|}
\hline & WBS & Controls & $\mathbf{P}$ \\
\hline Subjects, number & 41 & 205 & - \\
\hline Male:female ratio & $20: 21$ & $105: 100$ & - \\
\hline \multicolumn{4}{|l|}{ Age, yrs } \\
\hline Children & $9.6 \pm 2.74$ & $9.7 \pm 2.93$ & 0.861 \\
\hline Adults & $22.4 \pm 5.11$ & $22.3 \pm 5.42$ & 0.957 \\
\hline Height, SDS & & - & \\
\hline Children & $-0.7 \pm 0.60$ & $-0.1 \pm 0.81$ & $<0.001$ \\
\hline Adults & $-0.8 \pm 0.53$ & $-0.1 \pm 0.74$ & 0.006 \\
\hline \multicolumn{4}{|l|}{ BMI, SDS } \\
\hline Children & $-0.3 \pm 0.74$ & $0.1 \pm 0.62$ & 0.001 \\
\hline Adults & $-0.2 \pm 0.62$ & $0.2 \pm 0.81$ & 0.146 \\
\hline History of fracture, $\%$ & 12.2 & 10.2 & 0.505 \\
\hline Ad-SOS, z-score & $-1.4 \pm 1.39$ & $0.3 \pm 1.25$ & $<0.001$ \\
\hline Children & $-1.2 \pm 1.40$ & $0.0 \pm 0.81$ & $<0.001$ \\
\hline Adults & $-1.9 \pm 1.27$ & $-0.1 \pm 0.89$ & $<0.001$ \\
\hline BTT, z-score & $-0.8 \pm 1.15$ & $0.3 \pm 1.02$ & $<0.001$ \\
\hline Children & $-0.8 \pm 1.17$ & $0.3 \pm 0.98$ & $<0.001$ \\
\hline Adults & $-0.9 \pm 1.14$ & $0.2 \pm 0.74$ & $<0.001$ \\
\hline Calcium intake, mg/day & $760 \pm 239$ & $805 \pm 250$ & 0.290 \\
\hline Children & $778 \pm 252$ & $845 \pm 300$ & 0.246 \\
\hline Adults & $746 \pm 223$ & $789 \pm 201$ & 0.546 \\
\hline Vitamin D intake & $163 \pm 43$ & $178 \pm 47$ & 0.059 \\
\hline Children & $176 \pm 46$ & $194 \pm 51$ & 0.070 \\
\hline Adults & $153 \pm 40$ & $171 \pm 44$ & 0.236 \\
\hline Total calcium, mmol/L & $2.4 \pm 0.06$ & $2.2 \pm 0.16$ & $<0.001$ \\
\hline Ionised calcium, $\mathrm{mmol} / \mathrm{L}$ & $1.3 \pm 0.14$ & $1.1 \pm 0.13$ & $<0.001$ \\
\hline Phosphorus, mmol/L & $1.2 \pm 0.25$ & $1.3 \pm 0.22$ & 0.010 \\
\hline PTH, pg/mL & $51.7 \pm 17.74$ & $25.2 \pm 11.07$ & $<0.001$ \\
\hline BSAP, U/L & $100.9 \pm 29.83$ & $103.2 \pm 33.51$ & 0.683 \\
\hline Osteocalcin, ng/mL & $66.9 \pm 28.76$ & $91.0 \pm 23.62$ & $<0.001$ \\
\hline Urinary deoxypiridinoline, $\mathrm{nM} / \mathrm{mM}$ creatinine & $21.4 \pm 10.18$ & $40.2 \pm 15.61$ & $<0.001$ \\
\hline $25(\mathrm{OH}) \mathrm{D}, \mathrm{ng} / \mathrm{mL}$ & $23.7 \pm 7.28$ & $31.8 \pm 13.24$ & $<0.001$ \\
\hline $1,25(\mathrm{OH}) 2 \mathrm{D}, \mathrm{pg} / \mathrm{mL}$ & $83.2 \pm 17.64$ & $45.3 \pm 22.12$ & $<0.001$ \\
\hline
\end{tabular}

BMI: body mass index; 25(OH)D: 25-hydroxyvitamin D; 1,25(OH)2D: 1,25-dihydroxyvitamin D; PTH: parathyroid hormone 
the normal range in $12.2 \%$ of patients, with a mean of $83.2 \pm 17.64 \mathrm{pg} / \mathrm{mL}$ (controls: $45.3 \pm 22.12 \mathrm{pg} / \mathrm{mL}$; $\mathrm{p}<0.001)$. None of them exhibited low levels and 36 patients $(87.8 \%)$ had high $1,25[\mathrm{OH}] 2 \mathrm{D}$ levels.

A quantitative assessment of physical activity in patients with WBS and controls revealed significant differences between the two groups; the percentage of current physical activity levels was significantly lower for WBS patients compared with controls (0 hours per week group: $63 \%$ and $27 \%$, respectively; $<2$ hours per week group: $31 \%$ and $53 \%$, respectively; $>2$ hours per week group: $6 \%$ and $20 \%$, respectively).

Spearman's (rank) correlation test showed that AD-SoS z-score values were significantly inversely correlated with age for WBS patients $(r=-0.59$; $p$ $<0.005)$. Both BSAP and osteocalcin levels also showed a significant correlation with total calcium values $(r=0.49 ; p<0.005)$. PTH correlated significantly with ionised calcium $(\mathrm{r}=0.47 ; \mathrm{p}<0.05)$ and osteocalcin $(\mathrm{r}=0.68 ; \mathrm{p}<0.005)$.

The multiple regression analysis included age, sex, PTH, ionised and total calcium levels, phosphate levels, $25[\mathrm{OH}] \mathrm{D}$ and $1,25[\mathrm{OH}] 2 \mathrm{D}$ levels, serum osteocalcin levels, urinary deoxypyridinoline concentrations, quantitative assessment of physical activity, calcium intake, and BSAP levels; this analysis did not identify significant predictors of a lower AD-SoS z-score.

\section{DISCUSSION}

Our results confirm that WBS patients have an impaired bone mineral status and metabolism and we propose that this condition probably starts at the prepubertal age. Furthermore, nearly $80 \%$ of WBS patients with a positive history of fracture showed an impaired bone mineral status. This result underlines the importance of QUS evaluation in WBS starting as from childhood and adolescence for the early diagnosis of bone quality disorder.

QUS is in fact an easy-to-use, radiation-free, and portable technique that is particularly appropriate for assessing bone status in a long-term follow-up of paediatric syndromic patients with cognitive disabilities. $^{22}$

Although the International Society for Clinical
Densitometry (ISCD) 2007 Position Development Conference (PDC) stress the utility of QUS in fracture risk assessment, diagnosis of osteoporosis, treatment initiation and monitoring, and quality assurance/quality control, ${ }^{23}$ dual X-ray absorptiometry (DXA) is currently the most widely used tool for BMD measurement and must be considered as the gold standard for the diagnosis of osteoporosis and fracture prediction. ${ }^{24}$

Our data show that bone metabolism of WBS subjects appears to be altered and this impairment may be present in both paediatric and adult groups. Regarding calcium metabolism, the WBS patients analysed in our study showed increased calcium, PTH, and 1,25(OH)2D levels compared to healthy controls and this finding partially confirms the results of Cherniske et al. In fact, these authors reported a high percentage of increased PTH and 1,25(OH)2D levels and moderately reduced $25(\mathrm{OH}) \mathrm{D}$ levels in WBS adults. ${ }^{8}$ Unfortunately, they did not report the values of serum calcium, $25(\mathrm{OH}) \mathrm{D}, 1,25(\mathrm{OH}) 2 \mathrm{D}$, and PTH, nor those of dual X-ray absorptiometry to allow a comparison to be made. Interestingly, in the study of Cherniske et al, only WBS subjects over 35 years of age showed a reduction of the mean serum calcium levels and reduced osteocalcin levels. ${ }^{8}$ Our data seem to demonstrate a low bone formation in children and adolescents (i.e., reduced osteocalcin levels and normal deoxypyridinoline concentration); on the other hand, our adults showed increased bone resorption (i.e., normal osteocalcin levels and increased deoxypyridinoline concentration). These two markers may help to explain the impaired bone mineral status in WBS.

Aetiopathogenesis of bone metabolism disorder in WBS may be multifactorial, including both unknown bone characteristics specific to this genetic syndrome and environmental factors, i.e., lack of physical activity or poor nutrition. ${ }^{25-27}$ In fact, concerning lack of physical activity, WBS may show distinctive congenital and acquired cardiovascular lesions, thyroid disorders, and progressive joint limitations in addition to a variable failure to thrive during the infancy, observed in more than $70 \%$ of cases. ${ }^{1}$ Some subjects may develop coeliac disease. ${ }^{9}$ These findings are likely to variably contribute to the impaired bone mineral status in WBS and their identification may help in correcting modifiable risk factors to improve 
the bone health of these patients. Among these factors, the lower rate of motor activity among WBS patients is certainly of considerable importance our present study corroborating previous data on lack of physical activity in these patients. ${ }^{26}$ By contrast, WBS patients showed an intake of vitamin D and calcium comparable to that of the controls, which suggests that the problem may not be nutritional. Nevertheless, their 25(OH)D levels were significantly lower than those of controls, which could reflect reduced exposure to sunlight due to a different lifestyle. Many of the aspects highlighted by this cross-sectional study need to be confirmed and further substantiated with longitudinal data that would allow us to better grasp the effect of each variable on the metabolism and bone mineral status in WBS.

Regarding the causes of this apparent impairment of bone metabolism in patients with WBS, we may also hypothesise a dysregulation of calcium-PTH metabolism. In fact, calcium homeostasis is likely altered in patients with WBS due to infancy hypercalcaemia, hypercalciuria, or medullary nephrocalcinosis. ${ }^{28,29}$ However, when treated with low-calcium/ vitamin D-deficient infant formula, these patients developed rickets ${ }^{29}$ and osteoporosis in the first years of life ${ }^{30}$ despite increased 1,25(OH)2D levels. ${ }^{31}$ The mechanisms underlying transient hypercalcaemia in WBS patients are as yet poorly understood and there are currently several hypotheses that seek to explain this phenomenon. It is possible that hypercalcaemia depends on an increased renal sensitivity to PTH in normocalcemic WBS patients or that it depends on a reduced 1,25-dihydroxyvitamin D3 degradation. ${ }^{33,34}$ Furthermore, the deficiency of Williams syndrome transcription factor (WSTF), a nuclear protein codified by the Williams-Beuren Syndrome Chromosome Region 1 gene (WBSCR1, OMIM *603431), may play a role in the aetiology of hypercalcaemia in WBS, ${ }^{35}$ because of evidence of abnormal chromatin remodelling activity. ${ }^{36}$ Other authors suspect that the haploinsufficiency of the general transcription factor II-I gene (GTF2I, *601679) may have an effect on the impaired calcium metabolism. This gene encodes TFII-I, a multifunctional transcription factor ${ }^{37}$ that acts as a negative regulator of calcium entry by suppressing the surface accumulation of transient receptor potential C3 (TRPC3) channels. ${ }^{38}$ In the event of deficiency of GTF2I, TRPC3 seems to be over-expressed in the intestine and lymphocytes in WBS, potentially contributing to both gastrointestinal and renal calcium increased absorption. ${ }^{37}$ Moreover, other data appear to show a role of TFII-I as a potential negative regulator of osteoblast differentiation and of the Runx2-induced transcription of the osteocalcin gene, which is crucial to bone development. ${ }^{39}$ Thus, TFII-I haploinsufficiency may explain the impaired bone mineral status and bone metabolism data of WBS patients and down-regulation in both early (ALP) and late $(\mathrm{OCN})$ genetic markers of osteoblast differentiation. ${ }^{39}$

In conclusion, WBS patients exhibit a significant reduction in bone mineral status and are characterised by impaired bone metabolism. Further studies involving large series and conducted longitudinally will help us to better understand these dysregulations. We suggest close monitoring of bone mineral status and metabolism in WBS patients starting from the paediatric age. The role of possible treatment aimed to reduce these alterations will have to be assessed in the future.

\section{REFERENCES}

1. Pober BR, 2010 Williams-Beuren syndrome. N Engl J Med 362: 239-245.

2. Kaplan P, Wang PP, Francke U, 2001 Williams (Williams Beuren) syndrome: a distinct neurobehavioral disorder. J Child Neurol 16: 177-190.

3. Strømme P, Bjørnstad PG, Ramstad K, 2002 Prevalence estimation of Williams syndrome. J Child Neurol 17: 269-271.

4. Stagi S, Bindi G, Neri AS, et al, 2005 Thyroid function and morphology in patients affected by Williams syndrome. Clin Endocrinol (Oxf) 63: 456-460.

5. Stagi S, Lapi E, Cecchi C, et al, 2014 Williams-Beuren syndrome is a genetic disorder associated with impaired glucose tolerance and diabetes in childhood and adolescence: new insights from a longitudinal study. Horm Res Paediatr 82: 38-43.

6. Kruse K, Pankau R, Gosch A, Wohlfahrt K, 1992 Calcium metabolism in Williams-Beuren syndrome. J Pediatr 121: 902-907.

7. Jones KL, 1990 Williams syndrome: an historical perspective of its evolution, natural history, and etiology. Am J Med Genet Suppl 6: 89-96.

8. Cherniske EM, Carpenter TO, Klaiman C, et al, 2004 Multisystem study of 20 older adults with Williams syndrome. Am J Med Genet A 131: 255-264.

9. Stagi S, Lapi E, D’Avanzo MG, et al, 2014 Coeliac 
disease and risk for other autoimmune diseases in patients with Williams-Beuren syndrome. BMC Med Genet 15: 61 .

10. Stagi S, Lapi E, Gambineri E, et al, 2010 Bone density and metabolism in subjects with microdeletion of chromosome 22q11 (del22q11). Eur J Endocrinol 163: 329-337.

11. Stagi S, Pelosi P, Strano M, et al, 2014 Determinants of Vitamin D Levels in Italian Children and Adolescents: A Longitudinal Evaluation of Cholecalciferol Supplementation versus the Improvement of Factors Influencing 25(OH)D Status. Int J Endocrinol 2014: 58303.

12. Stagi S, Cavalli L, Bertini F, et al, 2014 Vitamin D levels in children, adolescents, and young adults with juvenile-onset systemic lupus erythematosus: a crosssectional study. Lupus 23: 1059-1065.

13. Holick MF, 2007 Vitamin D deficiency. N Engl J Med 357: 266-281.

14. Stagi S, Cavalli L, Signorini C, et al, 2014 Bone mass and quality in patients with juvenile idiopathic arthritis: longitudinal evaluation of bone-mass determinants by using dual-energy x-ray absorptiometry, peripheral quantitative computed tomography, and quantitative ultrasonography. Arthritis Res Ther 16: R83.

15. Drozdzowska B, Pluskiewicz W, Halaba Z, Misiolek H, Beck B, 2005 Quantitative ultrasound at the hand phalanges in 2850 females aged 7 to $77 \mathrm{yr}$ : a crosssectional study. J Clin Densitom 8: 216-221.

16. Pankau R, Partsch CJ, Gosch A, Oppermann HC, Wessel A, 1992 Statural growth in Williams-Beuren syndrome. Eur J Pediatr 151: 751-755.

17. Pankau R, Partsch CJ, Neblung A, et al, 1994 Natural history of body mass index in Williams-Beuren syndrome. Am J Med Genet 52: 51-54.

18. Partsch CJ, Dreyer G, Gosch A, et al, 1999 Longitudinal evaluation of growth, puberty, and bone maturation in children with Williams syndrome. J Pediatr 134: 82-89.

19. Cacciari E, Milani S, Balsamo A, et al, 2006 Italian cross-sectional growth charts for height, weight and BMI (2 to 20 yr). J Endocrinol Invest 29: 581-593.

20. Stagi S, Galli L, Cecchi C, et al, 2010 Final height in patients perinatally infected with the human immunodeficiency virus. Horm Res Paediatr 74: 165-1071.

21. Tanner JM, Whitehouse RH, 1976 Clinical longitudinal standards for height, weight, height velocity, weight velocity, and stages of puberty. Arch Dis Child 51: 170-179.

22. Stagi S, Cavalli L, Iurato C, et al, 2013 Bone health in children and adolescents: the available imaging techniques. Clin Cases Miner Bone Metab 10: 166-171.

23. Krieg MA, Barkmann R, Gonnelli S, et al, 2008 Quantitative ultrasound in the management of osteoporosis: the 2007 ISCD Official Positions. J Clin Densitom 11: 163-187.

24. Chin KY, Ima-Nirwana S, 2013 Calcaneal quantitative ultrasound as a determinant of bone health status: what properties of bone does it reflect? Int J Med Sci 10: 1778-1783.

25. Hocking DR, McGinley JL, Moss SA, et al, 2010 Effects of external and internal cues on gait function in Williams syndrome. J Neurol Sci 291: 57-63.

26. Nordstrøm M, Hansen BH, Paus B, Kolset SO, 2013 Accelerometer-determined physical activity and walking capacity in persons with Down syndrome, Williams syndrome and Prader-Willi syndrome. Res Dev Disabil 34: 4395-4403.

27. Nordstrøm M, Paus B, Andersen LF, Kolset SO, 2015 Dietary aspects related to health and obesity in Williams syndrome, Down syndrome, and Prader-Willi syndrome. Food Nutr Res 59: 25487.

28. Culler FL, Jones KL, Deftos LJ, 1985 Imparied calcitonin secretion in patients with Williams syndrome. J Pediatr 107: 720-723.

29. Mathias RS, 2000 Rickets in an infant with Williams syndrome. Pediatr Nephrol 14: 489-492.

30. Sangun O, Dundar BN, Erdogan E, 2011 Severe hypercalcemia associated with Williams syndrome successfully treated with pamidronate infusion therapy. J Pediatr Endocrinol Metab 24: 69-70.

31. Knudtzon J, Aksnes L, Akslen LA, Aarskog D, 1987 Elevated 1,25-dihydroxyvitamin D and normocalcaemia in presumed familial Williams syndrome. Clin Genet 32: 369-374.

32. Pronicka E, Rowińska E, Kulczycka H, et al, 1997 Persistent hypercalciuria and elevated 25-hydroxyvitamin D3 in children with infantile hypercalcaemia. Pediatr Nephrol 11: 2-6.

33. Lameris AL, Geesing CL, Hoenderop JG, Schreuder MF, 2014 Importance of dietary calcium and vitamin D in the treatment of hypercalcaemia in Williams-Beuren syndrome. J Pediatr Endocrinol Metab 27: 757-761.

34. Kruse K, Pankau R, Gosch A, Wohlfahrt K, 1992 Calcium metabolism in Williams-Beuren syndrome. J Pediatr 121: 902-907.

35. Barnett C, Krebs JE, 2011 WSTF does it all: a multifunctional protein in transcription, repair, and replication. Biochem Cell Biol 89: 12-23.

36. Kitagawa H, Fujiki R, Yoshimura K, Oya H, Kato S, 2011 Williams syndrome is an epigenome-regulator disease. Endocr J 58: 77-85.

37. Letavernier E, Rodenas A, Guerrot D, Haymann JP, 2012 Williams-Beuren syndrome hypercalcemia: is TRPC3 a novel mediator in calcium homeostasis? Pediatrics 129: e1626-e1630.

38. Caraveo G, van Rossum DB, Patterson RL, Snyder SH, Desiderio S, 2006 Action of TFII-I outside the nucleus as an inhibitor of agonist-induced calcium entry. Science 314: 122-125.

39. Lazebnik MB, Tussie-Luna MI, Hinds PW, Roy AL, 2009 Williams-Beuren syndrome-associated transcription factor TFII-I regulates osteogenic marker genes. J Biol Chem 284: 36234-36239. 